\title{
ARTICLE
}

\section{Development of a 3D human body library based on polygonal mesh surface for whole body counter set-up calibration}

\author{
Telma C. F. Fonseca ${ }^{\mathrm{a}^{*}}$, Anne Laure Lebacq ${ }^{\mathrm{a}}$, Liviu-Cristian Mihailescu ${ }^{\mathrm{a}}$, Filip Vanhavere ${ }^{\mathrm{a}}$ and Ria Bogaerts ${ }^{\mathrm{b}}$ \\ ${ }^{a}$ Belgian Nuclear Research Centre (SCK-CEN), Boeretang, 200 - 2400 Mol, Belgium; ${ }^{b}$ University Hospital Gasthuisberg, \\ Department of Radiation Oncology, Herestraat 49, B-3000, Leuven, Belgium
}

\begin{abstract}
A Whole Body Counter (WBC) is a common facility to routinely assess the internal contamination of exposed workers and especially in the case of radiation release accidents. The calibration of the counting device is usually done by using anthropomorphic physical phantoms representing the human body. Due to such a challenge of having representative physical phantoms a virtual calibration has been introduced. The use of computational phantoms and Monte Carlo methods to simulate radiation transport have been demonstrated to be a worthy alternative, not only as a complementary calibration method but also as a powerful tool for the design and optimization of counting geometries. In this study we introduce a methodology for building a 3D human body library that spans variation in both subject shape and size. Open source codes as MakeHuman and Blender software packages have been used for the creation and modelling of 3D humanoid characters based on polygonal mesh surfaces. Also, a home-made software was developed whose main goal is to convert the binary 3D voxel grid into a MCNPX input file. This paper summarizes (1) the development of the MaMP and FeMP - Male and Female Mesh Phantoms, the two first mesh phantoms generated from a series of 3D human bodies library created at SCK-CEN and (2) presents a comparison between the counting efficiency results obtained from the simulations of both mesh phantoms using MCNPX code.
\end{abstract}

Keywords: Monte Carlo; WBC; calibration; voxel phantoms; mesh geometry surface; computational phantoms; counting efficiency

\section{Introduction}

Whole Body Counter (WBC) is an appropriate set-up to measure the radiation emitted by individuals who are internally contaminated. The assessment of radioactive body burden requires calibration of the counting system which is usually done by using anthropomorphic physical phantoms representing the human body. However, due to large subject-specific variations in the measurement conditions, the calibration set-up can differ from the real measurement set-up to a great extent. The widespread availability of high performance computing has stimulated the research community to use computational phantoms and Monte Carlo methods to simulate radiation transport which has been demonstrated to be a worthy alternative, not only as a complementary calibration method but also as a powerful tool for the design and optimization of counting geometries, the analysis of uncertainties and the generation of information not directly obtainable from experimental methods. Variation of anatomical parameters such as the morphology aspects, the detector positioning relative to the individual and the

*Corresponding author. Email: tcff01@gmail.com uncertainties in the attenuation coefficients of the body can now be simulated easily resulting in different efficiency calibration curves.

Implementing geometries for particle transport problems is one of the major time consuming tasks. The common approach of radiation transport codes is based on Constructive Solid Geometry (CSG), this method uses simple objects usually called "solids" or "primitives", and allows a modeler to create a new solid object using boolean operators to combine very simple mathematical expressions to a complex geometry. Another geometry method for representing objects is the boundary representation (BREP). The polygonal mesh geometry representation is essentially a representation of solids by providing a relationship between vertices, edges, and surfaces. And the non-uniform rational basis spline (NURBS) geometry offers a common mathematical framework for implicit and parametric polynomial forms. This mathematical modelling tool is frequently used in computer graphics and animated films industry, thereby showing a method how to overcome some limitations related to the computational voxel phantoms [1-4]. Although the computational voxel phantoms are anatomically realistic models, most of them are made in supine position and due to the 
geometry modelling technique the resize of the voxel grids are the only operations allowed which introduces problems of smoothness and continuity when it is necessary to have these models in sitting position.

At SCK-CEN, the Belgian Nuclear Research Centre, a new method has been applied for the development of a new 3D human body library that spans variation in both subject shape and size. The MaMP (Male Mesh Phantoms) and FeMP (Female Mesh Phantoms) are the two first mesh phantoms generated from a series of 3D human body library and they have been used with monte carlo code for estimation and improvement of the counting efficiency of the WBC laboratory of the Nuclear Power Plant (NPP) in Doel, Belgium. Software packages as MakeHuman [5], Blender [6] and Binvox [7] have been used for the development and voxelization of the model and a home-made code named BIN-MCNPX was developed to convert the binary 3D voxel grid into a MCNPX [8] input deck file.

For the efficiency calibration of a WBC system the anthropometric parameters play an important role and in principle the internal organs are not needed. Instead, the body contour, shape and size are our main objects of study. The newly created 3D human body library is based on anthropometric information typically encountered in a WBC database of workers registered at SCK-CEN. This paper summarizes the procedure used for the development of the MaMP and FeMP Mesh Phantoms which are part of a 3D human body library based on polygonal Mesh surface and presents as well a comparison between the counting efficiency results obtained from the simulations of both mesh phantoms.

\section{Material and methods}

Figure 1 shows a schematic view of the essential steps taken to create MaMP and FeMP mesh phantoms.

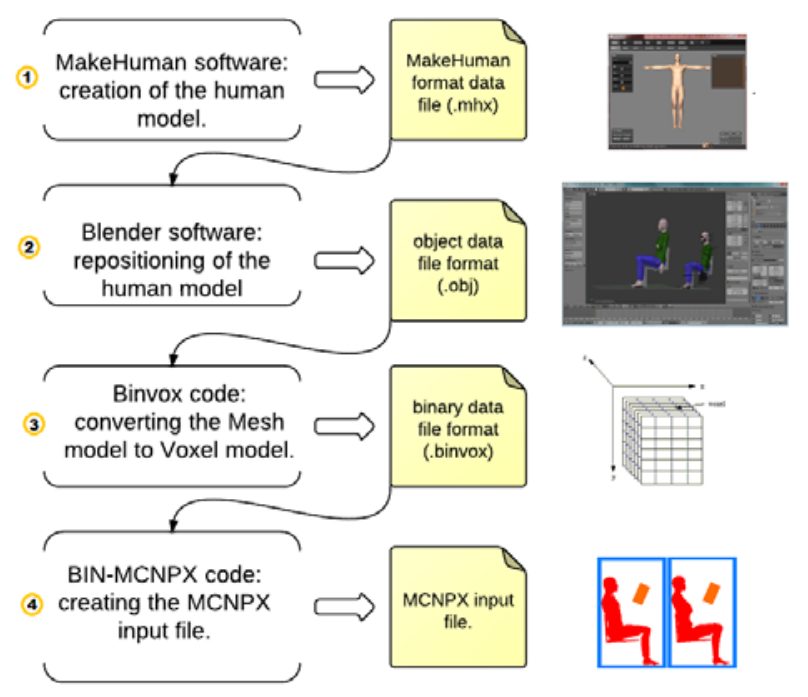

Figure 1. Schematic view of the essential steps taken to create MaMP and FeMP mesh phantoms.

\subsection{Creating male and female mesh phantoms}

The first step in creating the MaMP and FeMP mesh phantoms takes place in MakeHuman software. MakeHuman software is an open source application capable of realistically modelling a very wide variety of human anatomical forms in the full range of natural human poses from a single, universal mesh. The software is rather user-friendly and the model can be modeled using slider controls that combine different attributes like gender, age, muscles, weight, height breast size and firmness and face age etc. It takes into account the anthropometry parameters for modelling a character, for instance, if a female model is being modelled then any body mass added to the model will accumulate fat in those areas of the body where a woman typically accumulates fat. Figure $\mathbf{2}$ shows the MaMP and FeMP mesh phantoms and as example a small part of the female phantom showing the mesh structure in MakeHuman 1.0 Alpha 6.0 version.

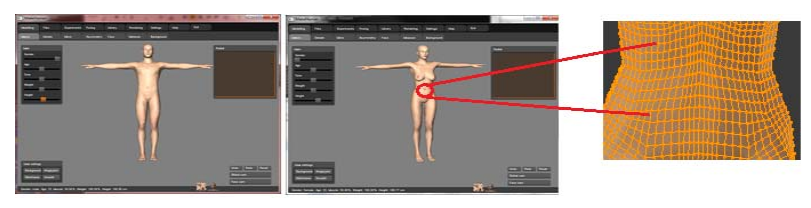

Figure 2. The MaMP and FeMP mesh phantoms and a small part of the mesh structure in MakeHuman software.

MakeHuman provides an enumerated list of file formats to export the data based on Mesh surfaces into a format desired; among them there is a "Blender exchange (mhx)" format data file. The .mhx file format enables to extract together with the Mesh Model the bony structures which are used later on for the free form deformation of the model, for example to bend the hips and legs into a sitting position, since they are still in upright position.

Once the 3D Mesh Model is finished, it needs to be exported to a format file handled by Blender software, this is where the second step begins. Blender is an open source 3D graphics application which provides modelling, texturing, lighting, animation and video post-processing functionality. Blender takes into account kinematics equations to determine as real as possible the movement of the human body into the mesh object. As an example, let's consider a human arm, the wrist has 2 DoF (degree of freedom), it can bend in any direction, but it cannot twist. The elbow also has 2 DoF: it can twist, and bend in one direction. Finally, the shoulder has 3 DoF.

Modification in the model can easily be done in any part of the body by moving the control points of polygonal mesh wireframes or simply moving a hoop or ring around the torso and a hoop or ring with arrows around the waist.

The "Blender exchange (.mhx)" data file format which contains the Mesh Model is imported into Blender and the desired modifications can be applied. For the WBC set-up normally the subject is sitting on a chair. 
The phantom's upper legs need to be bent at approximately $90^{\circ}$ angle related to the torso and the lower legs need to be bent at approximately $90^{\circ}$ angle related to the upper legs.

The third step starts by converting the Mesh Models into a voxelized phantom that can be read by MCNPX code and be used to perform the simulation task. For the voxelization process a straight forward program called Binvox was used. Binvox is a freeware 3D model converter which can convert the object data format of the 3D mesh geometry into a binary voxelized data file format. The object data file format stores the 3D geometry as vertex positions and normals. The faces that make each polygon are defined as a list of vertices. The binvox data file format stores a 3D voxel lattice assigning different numbers for the humanoid and for the universe which contains the humanoid.

Binvox code can process up to 1024 voxels per matrix dimension, though for the voxelization of the MaMP and FeMP mesh phantoms 256 voxels per matrix dimension were chosen. The resulting cubic voxel resolution is $0.53 \mathrm{~cm}$ for a $180 \mathrm{~cm}$ length phantom which was enough for keeping the smoothness of all mesh models. For means of internal dosimetry, when the calculation of the dose in small parts of the organ or tissue is required, the resolution of the computational phantom plays an important role. However, for counting efficiency calculations such high resolutions of the phantom are not required. The voxelization process is relatively inexpensive in computation time, which is approximately 5 minutes using a CoreI7 $1.73 \mathrm{GHz}, 8$ GB of memory and a FX880M card device.

One additional issue at that point of the whole process was to find a way to match the coordinate systems for the three codes: Blender, Binvox and MCNPX. For solving this issue a chair bent at an angle of $7^{\circ}$ degrees from the horizontal reference plane corresponding to the size and shape of the chair of the real WBC set-up, was drawn together with the humanoid into Blender. The purpose of this was to obtain some reference points to be sure that any humanoid would always be seated at the same position with respect to the chair.

As a fourth step, a home-made straight forward program named BIN-MCNPX was developed in $\mathrm{C}$ programming language [9] whose purpose is to read the binary data file resulting from the binvox code and to create a MCNPX input file which contains the 3D voxel lattice of the phantom. The $3 \mathrm{D}$ voxel lattice is read into a MCNPX input file which contains the chamber, the HPGe detector, the material composition, the densities and the source card configuration.

The phantom weight is obtained by multiplying the mass per a voxel by the total number of phantom voxels. The vertical length of voxel size is obtained from a relative calculation which takes into account the height of a reference individual body in sitting position. The height of a reference individual body in sitting position is the summing of the crown-buttock and the popliteal heights. Taking a $180 \mathrm{~cm}$ individual it gives as result $136 \mathrm{~cm}$ from bottom (feet) to top of the head. These two parameters were commonly used in the project DINBelg 2005 [10] which stores tables of anthropometric values for the Belgian population. These tables were used to estimate the body dimensions of the Flemish population.

The vertical length of voxel is calculated by multiplying the height of the reference individual body in sitting position $(136 \mathrm{~cm})$ by the height of the new phantom $(\mathrm{H} \mathrm{cm})$ and dividing it by the height of the reference individual body $(180 \mathrm{~cm})$ and finally dividing it by the resolution of the model (256), as showed in Eq. 1. For each new phantom height the voxel size needs to be calculated and changed manually in the MCNPX input file.

$$
\text { voxel }=\frac{(136 \times H) / 180}{256}
$$

\subsection{Creating the 3D human body library}

The WBC database of people monitored at the AGM laboratory of SCK-CEN in Mol, Belgium has registered a total of 894 individuals, 291 females and 603 males with age average of 41 years and 38 years old for male and female respectively, varying from $150 \mathrm{~cm}$ to $200 \mathrm{~cm}$ length. These individuals represent the most common population monitored at the Flemish region in Belgium. This database was used as reference data for building the 3D human body library. The different phantom configurations were selected following a sampling strategy by height and weight. For each height the mean value and the two extreme (maximum and minimum) weight values were chosen to limit the number of selected individuals while trying to keep a satisfying sampling. Applying this selection conditions, 28 phantoms were defined. In order to cover the possible weight values without exceed the difference of $50 \mathrm{~kg}$ between two phantoms, 1 new male phantom had to be defined. And 7 new female phantoms had to be defined varying randomly at different breast sizes. A total of 36 different mesh phantoms, 14 male mesh phantoms varying from $160 \mathrm{~cm}$ to $200 \mathrm{~cm}$ length and 22 female mesh phantoms varying from $150 \mathrm{~cm}$ to $190 \mathrm{~cm}$ length have been created.

\subsection{MCNPX Simulation}

The general idea of Monte Carlo analysis is to create a model which is as close as possible to the real system of interest, and to create interactions within that system based on known probabilities of occurrence, with random sampling of the probability density functions. The Monte Carlo code is usually used to simulate radiation transport, taking into account the relevant photon interaction processes (the photoelectric absorption, Compton scattering, Rayleigh scattering and electron-positron pair production).

The simulations were performed with MCNPX Code version 2.7.d running under MPI (Multiprocessor) in a Computational Cluster of SCK-CEN. The MCNPX code was used in conjunction with the photonuclear library (plib04), based on the ENDF/B-VII. In each run up to 
$10^{8}$ particle histories were tracked to achieve good statistics, less than $1 \%$. MCNPX reports estimated statistical uncertainties in terms of fractional standard deviations, namely the relative errors. Approximately $3 \mathrm{~h}$ of running time was required in the cluster with 4 nodes and 16 cpu's slaves of $2.4 \mathrm{GHz}$ CoreI7 hyperthreaded and 32 GB of memory, installed under a CentOS linux operational system.

The Monte Carlo simulations have been performed for MaMP and FeMP mesh phantoms filled with ${ }^{241} \mathrm{Am}$, ${ }^{133} \mathrm{Ba},{ }^{60} \mathrm{Co},{ }^{137} \mathrm{Cs}$ and ${ }^{152} \mathrm{Eu}$. Since there are no internal organs the sources were homogenously distributed in the phantom. It is planned to extend the home-made code by implementing new capabilities which allows adding the internal organs in the mesh phantom for purpose of lung counting systems.

The specific MCNPX tally, named F8, to record the pulse-height spectrum was used. The source card was set-up per gamma-ray energy. This means that for each gamma-ray energy a MCNPX input file was needed, resulting in a total of 14 input files to cover the gamma-ray energy range between $59.4 \mathrm{keV}$ to $1408 \mathrm{keV}$. A software tool called mcnp_pstudy [11] has been used to automate the creation of the input files from a series of MCNPX calculations varying the energy card.

\section{Results and discussion}

Figure 3 shows the collection of female and male phantoms varying in shapes and sizes created to compose the 3D human body library based on polygon mesh geometry. Although these phantoms are shown in supine position, they were simulated in sit position.
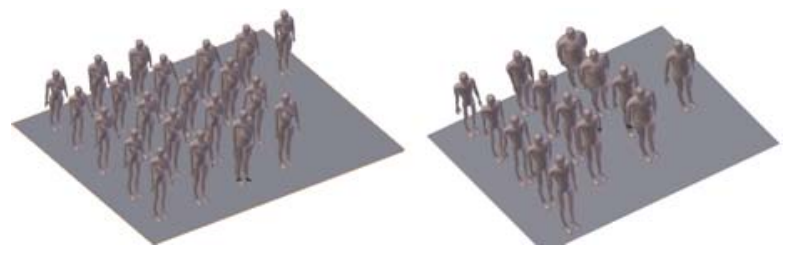

Figure 3. Collection of female and male mesh phantoms varying in shapes and sizes.

\subsection{Counting efficiencies for male and female mesh phantoms}

The MaMP and FeMP mesh phantom were used for the Whole Body Counter (WBC) laboratory of the Nuclear Power Plant (NPP) in Doel, Belgium. These two first phantoms created from a series of 3D human body library have a height of $180.60 \mathrm{~cm}$ and $180.77 \mathrm{~cm}$ and a weight of $87.6 \mathrm{~kg}$ and $84.5 \mathrm{~kg}$ for male and female phantoms respectively. Since there are no internal organs defined in the voxel phantom the material composition in the body was taken to be homogenous mixture of two different compounds - 50th percent of both muscles and soft tissue - and the density was taken to be $1.05 \mathrm{~g} \mathrm{~cm}^{-3}$.

The anthropometric data was obtained from the reference elemental compositions given in ICRP Publication 110 [12] for muscle tissue and for soft-tissue the elemental compositions were taken from Kramer et all [13]. Table 1 shows the elemental composition for MaMP and FeMP mesh phantoms.

Table 1. Elemental compositions of muscle and soft tissue for the MaMP and FeMP phantoms.

\begin{tabular}{lcc}
\hline $\begin{array}{l}\text { Element } \\
\text { composition }\end{array}$ & $\begin{array}{c}\text { Muscle } \\
\text { (\% by mass) }\end{array}$ & $\begin{array}{c}\text { Soft Tissue } \\
\text { (\% by mass) }\end{array}$ \\
\hline $\mathrm{H}_{1}$ & 10.20 & 10.50 \\
$\mathrm{C}_{6}$ & 14.20 & 12.50 \\
$\mathrm{~N}_{7}$ & 3.40 & 2.60 \\
$\mathrm{O}_{8}$ & 71.10 & 73.50 \\
$\mathrm{Na}_{11}$ & 0.10 & 0.20 \\
$\mathrm{P}_{15}$ & 0.20 & 0.20 \\
$\mathrm{~S}_{16}$ & 0.30 & 0.18 \\
$\mathrm{Cl}_{17}$ & 0.10 & 0.22 \\
$\mathrm{~K}_{19}$ & 0.40 & 0.21 \\
$\mathrm{Ca}_{20}$ & & 0.01 \\
$\mathrm{Fe}_{26}$ & & 0.01 \\
\hline
\end{tabular}

The YZ axis cross-sectional view of the MaMP and FeMP - Male and Female Mesh Phantoms and the HPGe detector implemented in MCNPX are shown in Figure 4.

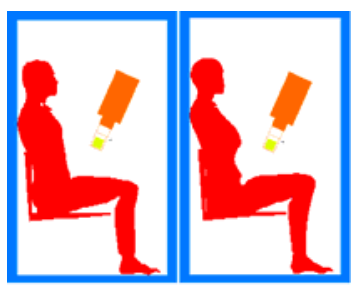

Figure 4. The MaMP and FeMP mesh phantoms in the bunker with an HPGe detector. (no scale)

Table 2 shows the counting efficiency results simulated with MaMP - Male Mesh Phantom and FeMP - Female Mesh Phantom and the ratios between both efficiency results. MaMP mesh phantom is heavier than FeMP so, the counting efficiency of FeMP mesh phantom is systematically higher with about $4 \%$ for all simulated energies compared with MaMP mesh phantom.

It is clearly observed that the body size and shape induce significant differences in the radiation attenuation for whole body counter set-up calibration. One may see that the counting efficiency decreases as body mass increases and it does vary for gender. Comparing both phantoms, they have same height and the source is homogenously distributed in the phantom, the only parameter which differs for both phantoms is the breast of the female phantom which is slightly closer to the HPGe detector compared with the MaMP phantom. It is also important to point out that due to the size of the breast the absorption of the gamma-ray in this region is not leading to a decrease of the counting efficiencies at low gamma-ray energies. The relative errors of the simulations are below $1 \%$ for all gamma-ray energy range at all simulated distances. 
Table 2. Counting efficiency simulated values for MaMP and FeMP phantoms and HPGe detector.

\begin{tabular}{lcccccc}
\hline Source & $\begin{array}{c}\text { Energy } \\
(\mathrm{keV})\end{array}$ & $\begin{array}{c}\text { Efficiency } \\
\text { MaMP }\end{array}$ & $\begin{array}{c}\text { Rel. } \\
\text { Error } \\
(\%)\end{array}$ & $\begin{array}{c}\text { Efficiency } \\
\text { FeMP }\end{array}$ & $\begin{array}{c}\text { Rel. } \\
\text { Error } \\
(\%)\end{array}$ & $\begin{array}{c}\text { Ratio } \\
\text { FeMP/ } \\
\text { MaMP }\end{array}$ \\
\hline $\mathrm{Am}_{241}$ & 59.5 & $1.67 \mathrm{E}-04$ & 0.8 & $1.73 \mathrm{E}-04$ & 0.8 & 1.03 \\
$\mathrm{Ba}_{133}$ & 81.0 & $3.26 \mathrm{E}-04$ & 0.6 & $3.40 \mathrm{E}-04$ & 0.5 & 1.04 \\
$\mathrm{Eu}_{152}$ & 121.8 & $4.56 \mathrm{E}-04$ & 0.5 & $4.75 \mathrm{E}-04$ & 0.5 & 1.04 \\
$\mathrm{Eu}_{152}$ & 244.7 & $4.06 \mathrm{E}-04$ & 0.5 & $4.25 \mathrm{E}-04$ & 0.5 & 1.05 \\
$\mathrm{Ba}_{133}$ & 302.9 & $3.64 \mathrm{E}-04$ & 0.5 & $3.79 \mathrm{E}-04$ & 0.5 & 1.04 \\
$\mathrm{Eu}_{152}$ & 344.3 & $3.38 \mathrm{E}-04$ & 0.5 & $3.52 \mathrm{E}-04$ & 0.5 & 1.04 \\
$\mathrm{Ba}_{133}$ & 356.0 & $3.32 \mathrm{E}-04$ & 0.6 & $3.45 \mathrm{E}-04$ & 0.6 & 1.04 \\
$\mathrm{Cs}_{137}$ & 661.7 & $2.36 \mathrm{E}-04$ & 0.7 & $2.44 \mathrm{E}-04$ & 0.7 & 1.03 \\
$\mathrm{Eu}_{152}$ & 778.9 & $2.17 \mathrm{E}-04$ & 0.7 & $2.24 \mathrm{E}-04$ & 0.7 & 1.03 \\
$\mathrm{Eu}_{152}$ & 964.1 & $1.94 \mathrm{E}-04$ & 0.7 & $2.01 \mathrm{E}-04$ & 0.7 & 1.04 \\
$\mathrm{Eu}_{152}$ & 1112.1 & $1.82 \mathrm{E}-04$ & 0.7 & $1.91 \mathrm{E}-04$ & 0.7 & 1.05 \\
$\mathrm{Co}_{60}$ & 1173.2 & $1.77 \mathrm{E}-04$ & 0.8 & $1.83 \mathrm{E}-04$ & 0.7 & 1.03 \\
$\mathrm{Co}_{60}$ & 1332.5 & $1.65 \mathrm{E}-04$ & 0.8 & $1.71 \mathrm{E}-04$ & 0.8 & 1.04 \\
$\mathrm{Eu}_{152}$ & 1408.0 & $1.60 \mathrm{E}-04$ & 0.8 & $1.66 \mathrm{E}-04$ & 0.8 & 1.04 \\
\hline
\end{tabular}

\section{Conclusion}

We have presented a methodology to create the MaMP (Male Mesh Phantom) and FeMP (Female Mesh Phantom) and a comparison of the counting efficiency results between these two mesh phantoms developed for the WBC laboratory of KCDoel in Belgium. The proposed method for the development of mesh phantoms uses the free open source codes MakeHuman and Blender for modelling and deforming the mesh object. The voxelization process is done by using a straight forward freeware code called Binvox. And a home-made code named BIN-MCNPX was developed for converting the binary 3D voxel grid data into MCNPX input file which contains the 3D voxel lattice of the phantom.

The advantage of using computational phantoms to calibrate in vivo systems lies in that it is relatively easy to modify the position of the phantom with respect to the detector, the shape and size of the phantom, the material composition and density, etc. A total of 36 different mesh phantoms varying in length and weight have been created. These phantoms are the first created with no internal organs with a specific purpose of studying the morphology-induced variation on the counting efficiency of a counting system.

\section{Acknowledgements}

The author gratefully acknowledges E. Malambu from SCK-CEN and R. Wyckmans and L. Claessens from NPP in Doel for fruitful discussion of the topics.

\section{References}

[1] X. G. Xu and K. F. Eckerman, Handbook of Anatomical Models for Radiation Dosimetry, Taylor \& Francis (2009), ISBN 9781-4200-5980-9.

[2] C. Lee, J. Hurtado, J. L. Williams and W. E. Bolch, Whole-body voxel phantoms of paediatric patients - UF Series B, Phys. Med. Biol. 51 (2006), pp. 4649-4661.

[3] C. Lee, D. Lodwick, J. Hurtado, J. L. Williams and W. E. Bolch, The UF family of reference hybrid phantoms for computational radiation dosimetry, Phys. Med. Biol. 55 (2) (2009), pp. 339-363.

[4] J. Farah, D. Broggio and D. Franck, Creation and use of adjustable 3D phantoms: Applicaion for the lung monitoring of female worker, Health Physics 99 (5) (2010), ISSN 0017-9078.

[5] M. Bastioni, The MakeHuman ${ }^{\mathrm{TM}}$ Application Open source tool for making 3D characters, http://www.makehuman.org/blog/index.php, date last accessed in November 2011.

[6] Blender.org, Blender - free open source 3D content creation suite. http://www.blender.org/, last accessed in November (2011).

[7] M. Patrick, Binvox code - 3D mesh voxelizer, http://www.patrickmin.com/minecraft/, last accessed in November (2011).

[8] D. B. Pelowitz, M. R. James, G. W. McKinney, J. W. Durkee, M. L. Fensin, J. S. Hendricks, S. G. Mashnik, J. M. Verbeke and L. S. Waters, MCNPX 2.7.D extensions, LA-UR-10-07031, Los Alamos National Security, (2010).

[9] M.D. Ritchie, http://cm.bell-labs.com/cm/cs/who/dmr/, last accessed in December (2012).

[10] R. Motmans, Ergonomie RC 2005 DINBelg Ergonomie RC, Assoc. KULeuven in Leuven Belgium, (2006)

http://www.dinbelg.be/anthropometry.htm.

[11] B. B. Forrest, E. S. Jeremy and R. Hayes, Monte carlo parameter studies and uncertainty analyses with MCNP5. Vol 452 (2004), Los Alamos National Laboratory, pp. 452-509.

[12] ICRP Publication 110, Adult Reference Computational Phantoms, Annals of the ICPR Volume 39 Issue 2, 1e, International Commission on Radiological Protection, (2009), ISBN-10: 0702041866.

[13] R. Kramer, J. W. Vieira, H. J. Khoury and E. C. M. Loureiro, Comparison of effective dose between tomographic and mathematical phantoms for external exposures to photons, Proc. The $11^{\text {th }}$ Int. Congress of the International Radiation Protection Association, Madrid, Spain, May 23-28, (2004). 